\title{
Clinical outcomes of vitrified-thawed embryo transfer using a pull and cut straw method
}

\author{
Joon Gyo Lim ${ }^{1,2^{*}}$, Young Tae Heo ${ }^{1 *}$, Seung Gi Min ${ }^{3}$, Byeong Yeol Min², Sang Jun Uhm ${ }^{4}$ Nam Hyung Kim ${ }^{1}$ \\ ${ }^{1}$ Department of Animal Sciences, Chungbuk National University, Cheongju; ${ }^{2}$ Min Byeong Yeol Obstetrics and Gynecology, Cheongju; ${ }^{3} \mathrm{Chungbuk}$ \\ National University School of Medicine, Cheongju; ${ }^{4}$ Department of Animal Science and Biotechnology, Sangji Youngseo College, Wonju, Korea
}

\section{Objective}

To compare the clinical outcomes of patients with vitrified-thawed embryos transferred using either the $0.25 \mathrm{~mL}$ straw method and the pull and cut straw (PNC) method. To evaluate the clinical outcomes of patients with transferred embryos that underwent assisted hatching at the cleaved embryo (day 3) or the blastocyst (day 5) stage.

\section{Methods}

The study population consisted of women who underwent vitrified-warmed embryo transfer between May 2000 and December 2011 and assisted hatching was performed after warming of embryos. Cycles of thawing between assisted hatching treated and non treated groups were compared for survival and pregnancy rates.

\section{Results}

The PNC vitrification method improved survival and pregnancy rates in partial lysed embryos. While assisted hatching did not affect the developmental and clinical pregnancy rates of the vitrified-warmed blastocyst group, it did increase the pregnancy rate of poor quality vitrified-warmed cleaved embryos.

\section{Conclusion}

These results suggest that PNC may increase the number of clinical pregnancies via the vitrification of both cleaved embryos and blastocysts. In addition, selective assisted hatching treatment of embryos that show a poor prognosis after warming may increase the rate of clinical pregnancy.

Keywords: Assisted hatching; Pull and cut straw; Vitrified-thawed embryo

\section{Introduction}

Since the first ice-free cryopreservation of mouse embryos by vitrification [1], many studies have helped to simplify and shorten the cryopreservation procedure. The vitrification of human embryos has been extensively tested and is now a routine technique in assisted reproduction laboratories [2]. Among the variety of procedures used to cryopreserve embryos, a hand-made container called a pull and cut straw (PNC) has recently been reported [3]. Studies indicate that PNC is useful for the vitrification of human blastocysts and cleavagestage embryos. The technique also has the benefits of simplicity, cost-efficiency and rapid cooling [4].

It is well known that damage caused by freezing is an important factor influencing the quality of embryos. Such dam- age induces zona hardening, delays embryo development and perturbs zona pellucida morphology, reducing the ability of

Received: 2012.10.30. Revised: 2013.2.1. Accepted: 2013.3.18.

Corresponding author: Nam Hyung Kim

Department of Animal Sciences, Chungbuk National University, 52 Naesudong-ro, Heungdeok-gu, Cheongju 361-763, Korea Tel: +82-43-261-2546 Fax: +82-43-272-8853

E-mail: nhkim@chungbuk.ac.kr

*These authors contributed equally to this study.

Articles published in Obstet Gynecol Sci are open-access, distributed under the terms of the Creative Commons Attribution Non-Commercial License (http://creativecommons. org/licenses/by-nc/3.0/) which permits unrestricted non-commercial use, distribution, and reproduction in any medium, provided the original work is properly cited.

Copyright $\odot 2013$ Korean Society of Obstetrics and Gynecology 


\section{Obstetrics \& Gynecology Science}

Joon Gyo Lim, et al. Freezing and assisted hatching of embryo

embryos to hatch and implant [5-7]. Another cause of reducing the ability of embryos is transfer of partial lysed embryos [8]. A previous studies reported that clinical pregnancy and implantation rates were significantly increased after zona drilling of frozen embryos or removing of lysed blastomeres in vitrified and warmed embryos [9-11]. However, another study found that assisted hatching (AH) treatment of embryos from both human and animal models has no effects on embryo development and pregnancy [12]. Many in vitro fertilization (IVF) centers now perform $\mathrm{AH}$ procedures for infertility patients in the hope of increasing the chance of each embryo successfully hatching and implanting. Therefore, detailed investigations into the effects of $\mathrm{AH}$ on the embryo under specific conditions and/or developmental stages may offer valuable information to the human IVF/embryo transfer (ET) program.

In this study, we describe PNC and compare the rates of embryo survival and clinical pregnancy of vitrified-warmed embryos using PNC and conventional $0.25 \mathrm{~mL}$ straw containers. In addition, we investigated the pregnancy rate of vitrified-warmed human cleavage-stage embryos and blastocysts with $\mathrm{AH}$ treatment.

\section{Materials and methods}

\section{Patients}

Couples entered the IVF-ET program for infertility treatment either because of male factors (intracytoplasmic sperm injection [ICSI] with ejaculate, epididymal or testicular sperm) and/ or female factors (tubal, endometriosis or idiopathic). Vitrifiedwarmed embryos were transferred into women whose ages ranged from 23 to 46 years after being cultured in medium (Table 1). Vitrification was performed using supernumerary embryos that had reached cleaved embryo stage after 3 days and blastocyst stage after five days of culture.

\section{Superovulation and collection of oocytes}

Ovarian stimulation was performed using urinary folliclestimulating hormone (FSH, highly purified-FSH, Serono, Geneva, Switzerland), recombinant FSH (Gonal F, MerckSerono, Darmstadt, Germany) or hMG (Pergona, Serono, Switzerland; Merional, IBSA, Lugano, Switzerland) in combination with a gonadotropin-releasing hormone (GnRH) antagonist (Cetrotide, Merck-Serono, Germany) or agonist (Suprefact, Aventis Pharma, Frankfurt, Germany). Oocyte maturation was induced by injection of 10,000 IU human chorionic gonadotropin (hCG; IVF-C, LG, Seoul, Korea; choriomon, IBSA, Switzerland or Profasi, Merck-Serono, Geneva, Switzerland) as soon as three follicles with a size of $18 \mathrm{~mm}$ were observed by ultrasound. Oocytes were retrieved 36 hours after hCG administeration using vaginal ultrasoundguided puncture of ovarian follicles.

Table 1. Vitrification-warming of cleaved embryos and blastocysts using conventional straw and PNC

\begin{tabular}{|c|c|c|c|c|}
\hline & Straw-BL & PNC-BL & PNC-CL & PNC-Total \\
\hline No. of cycles for warming & 30 & 12 & 78 & 90 \\
\hline Age $(y r$ mean $\pm S D)$ & $31.3 \pm 3.8$ & $33 \pm 3.9$ & $32.5 \pm 4.5$ & $32.6 \pm 4.4$ \\
\hline Age range (yr) & $24-38$ & $23-37$ & $24-46$ & $23-46$ \\
\hline No. of embryos warmed & 88 & 41 & 518 & 559 \\
\hline No. of recovery embryos (\%) & $88(100)$ & $41(100)$ & $518(100)$ & $559(100)$ \\
\hline \multicolumn{5}{|l|}{ Embryo development after $17-18$ hours culture } \\
\hline No. of IE after warming (\%) & $63(71.6)$ & $31(75.6)$ & $316(61.0)$ & $347(62.1)$ \\
\hline No. of PL embryos (\%) & & & $183(35.3)$ & $183(32.7)$ \\
\hline No. of survival embryos (IE+PL) (\%) & $63(71.6)^{a)}$ & $31(75.6)^{a)}$ & $499(96.3)^{b)}$ & $539(94.8)$ \\
\hline No. of ET cycles & 27 & 12 & 78 & 90 \\
\hline No. of transferred embryos (mean \pm SD) & $62(2.3 \pm 1.1)$ & $29(2.4 \pm 0.7)$ & $349(4.5 \pm 1.7)$ & $378(4.2 \pm 1.8)$ \\
\hline $\begin{array}{l}\text { No. of clinical pregnancies (\% pregnancies/ } \\
\text { transfer) }\end{array}$ & $12(44.4)$ & $9(75.0)$ & $37(47.4)$ & $46(51.1)$ \\
\hline
\end{tabular}

PNC, pull and cut straw; Straw-BL, blastocyst vitrification with a conventional straw; PNC-BL, blastocyst vitrification with PNC; PNC-CL, cleaved embryo vitrification with PNC; SD, standard deviation; IE, intact embryos; PL, partial lysed; ET, embryo transfer.

Values with different superscripts $\left({ }^{a)}\right.$ and ${ }^{\text {b) }}$ ) differ significantly $(P<0.05)$. 


\title{
Obstetrics \& Gynecology Science
}

\author{
Vol. 56, No. 3, 2013
}
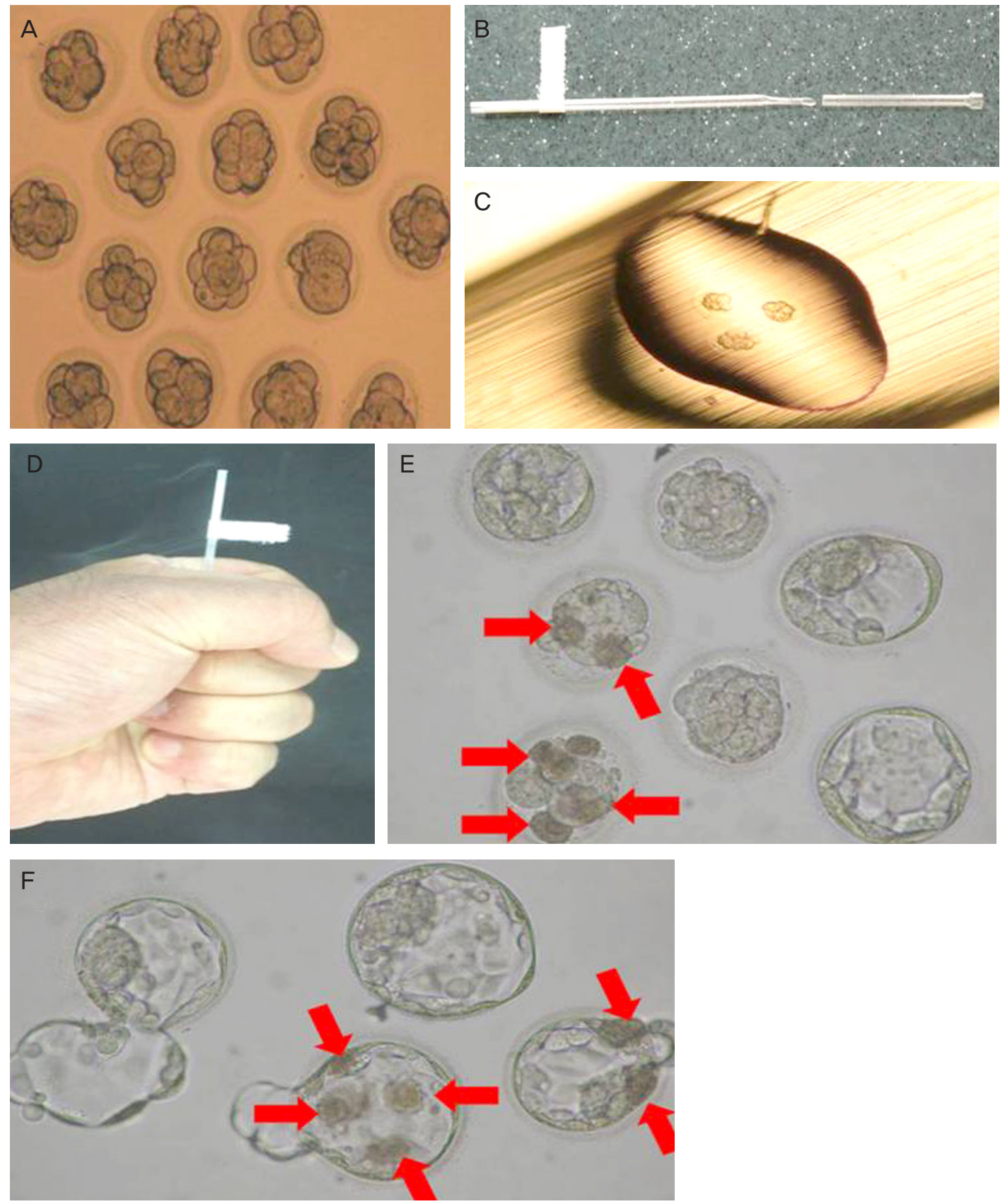

G

\begin{tabular}{|c|c|c|c|c|c|c|c|c|c|c|}
\hline Open & & Air & $\begin{array}{c}\text { VS } \\
\text { embryo }\end{array}$ & Air & VS & Air & $0.5 \mathrm{M} \mathrm{S}$ & Air & \begin{tabular}{|l|}
0.5 \\
$M S$
\end{tabular} & Cotten \\
\hline & $\begin{array}{l}0.3 \\
\mathrm{~cm}\end{array}$ & $\begin{array}{l}0.5 \\
\mathrm{~cm}\end{array}$ & $1 \mathrm{~cm}$ & $\begin{array}{l}0.5 \\
\mathrm{~cm}\end{array}$ & $\begin{array}{l}0.3 \\
\mathrm{~cm}\end{array}$ & $\begin{array}{l}0.5 \\
\mathrm{~cm}\end{array}$ & $6 \mathrm{~cm}$ & $\begin{array}{l}0.5 \\
\mathrm{~cm}\end{array}$ & $\begin{array}{l}0.5 \\
\mathrm{~cm}\end{array}$ & \\
\hline
\end{tabular}

Fig. 1. Vitrification-warming of embryos using the modified cut standard straw (PNC) method and structure of conventional straw. (A) Day 3 human cleaved embryos ( $\times 40)$. (B) Shape of the PNC ( $\times 40)$. (C) Loaded embryos on the tip of the PNC with a minimum volume of vitrification solution $(\times 40)$. (D) Warming of embryos in the PNC by holding the PNC with bare hands $(\times 40)$. (E) Cultured embryos for 42 hours after warming (arrows indicate partial lysed cell) $(\times 40)$. (F) Hatching embryos cultured for 66 hours after warming and assisted hatching (arrows indicate partially lysed cells) $(\times 40)$. (G) Structure of conventional straw for embryo vitrification (VS, vitrification solution; S, sucrose) $(\times 40)$. 


\section{Obstetrics \& Gynecology Science}

Joon Gyo Lim, et al. Freezing and assisted hatching of embryo

\section{Fertilization of oocytes and culture of zygotes}

Collected oocytes were cultured in P1 media (Irvine Scientific, Irvine, (A, USA) supplemented with 10\% synthetic serum substitute (SSS) (Irvine Scientific) for 3 to 4 hours. In preparation for ICSI, cumulus and corona cells were removed from the oocyte and the cumulus complex using HTF media (Irvine Scientific) supplemented with $100 \mathrm{IU} / \mathrm{mL}$ hyaluronidase (Type VIII, Sigma Chemical Co., St. Louis, MO, USA). Following ICSI, oocytes were cultured in P1 media supplemented with $10 \%$ SSS for 18 hours. The zygotes obtained by this procedure were selected on the basis of their morphology and formation of nucleus which showed two pronuclei and second polar body then were cultured for a further 48 hours. Embryos remaining after embryo transfer were vitrified or sequentially cultured in G2 Medium (Vitrolife AB, Kungsbacka, Sweden) for three days until they developed into blastocysts then vitrified.

\section{Vitrification of cleavage embryos and blastocysts}

Vitrification of embryos and blastocysts was performed using two containers: a PNC vessel and a conventional $0.25 \mathrm{~mL}$ straw [3]. During conventional $0.25 \mathrm{~mL}$ straw vitrification, blastocysts were incubated in 10\% glycerol in Dulbecco's phosphate buffered saline (DPBS, Gibco, Grans Island, NY, USA) for 5 minutes, transferred to $10 \%$ glycerol, $20 \%$ ethylene glycol (Sigma-Aldrich) in DPBS for 5 minutes and finally transferred to $25 \%$ glycerol, $25 \%$ ethylene glycol in DPBS for 1 minute. Blastocysts were then loaded as described by Schiewe et al. [13]. Briefly, the following components were placed in the following order into a $0.25 \mathrm{~mL}$ plastic straw: 6 $\mathrm{cm}$ column of $1 \mathrm{M}$ sucrose in DPBS; $0.5 \mathrm{~cm}$ air space; $0.5 \mathrm{~cm}$ of vitrification solution (25\% ethylene glycol, $25 \%$ glycerol, 0.5 $M$ sucrose and 10\% (v/v) SSS in DBPS); $0.5 \mathrm{~cm}$ air space; 1 $\mathrm{cm}$ of vitrification solution with embryos. The straw was then plunged into liquid nitrogen for storage (Fig. 1).

For the vitrification of cleavage embryos and blastocysts using PNC, embryos and blastocysts were incubated in 7.5\% ethylene glycol, 7.5\% DMSO (Sigma-Aldrich) and 10\% (v/ v) SSS in DBPS for 15 minutes and were then transferred to vitrification solution (15\% ethylene glycol, 15\% DMSO, $0.5 \mathrm{M}$ sucrose in DPBS) for 1 minute. Briefly, the PNC container was constructed by heating the middle part of a standard $0.25 \mathrm{~mL}$ insemination straw, which was then molded to achieve an inner diameter of $0.1 \mathrm{~mm}$ and then cut at a $45^{\circ}$ angle. Embryos and blastocysts were loaded onto the edge of the PNC in less than $1 \mu \mathrm{L}$ of vitrification solution and were then covered with the remaining part of the straw. The PNC was then plunged into liquid nitrogen for storage. Period of vitrification of blastocysts using conventional $0.25 \mathrm{~mL}$ straw was from May 2000 to March 2005 and its of PNC method was from April 2005 to December 2011. One to three embryos or blastocysts were loaded into each container.

\section{Warming of vitrified embryos}

For the PNC protocol, embryos were warmed by holding the container with bare hands $\left(33^{\circ} \mathrm{C}\right.$ to $\left.34^{\circ} \mathrm{C}\right)$ for 5 seconds. For the traditional protocol, straws were warmed in a $22^{\circ} \mathrm{C}$ water bath for 10 seconds. The post-warming steps were the same for both container types: the thawed embryos were sequentially transferred to a $1 \mathrm{M}$ sucrose solution for 1 minutes $\left(37^{\circ} \mathrm{C}\right)$, then to a $0.5 \mathrm{M}$ sucrose solution for 3 minutes $\left(25^{\circ} \mathrm{C}\right)$ and finally to $10 \%(\mathrm{v} / \mathrm{v}) \mathrm{SSS}$ in PBS for 10 minutes $\left(25^{\circ} \mathrm{C}\right)$. The survival of vitrified embryos following warming was evaluated on the basis of the re-expansion, transparency and shape of the cytoplasm after 18 to 24 hours.

\section{AH with acidified medium}

Cultured embryos underwent hatching in response to acidified medium delivered with a hatching micropipette after vitrification-warming procedures. $\mathrm{AH}$ was selectively performed on embryos with a poor prognosis (thick zona pellucida: $>15$ $\mu \mathrm{m}$, a retarded developmental rate and excessive fragmentation), or which showed low response for controlled ovarian hyperstimulation and had previously failed two or more IVF attempts.

Egg-holding and $\mathrm{AH}$ micropipettes were obtained from Humagen Fertility Diagnostics, Inc. (Charlottesville, VA, USA). During $A H$, embryos were rinsed and placed onto $0.1 \mathrm{~mL}$ droplets of modified HTF Medium HEPES (Irvine Scientific) supplemented with 10\% (v/v) SSS. Acidified Tyrode's solution (Irvine Scientific) was then used to generate an opening of approximately 10 to $15 \mu \mathrm{m}$ in diameter in the zona pellucida as previously described [14]. Embryos were then rinsed and placed in an embryo transfer dish containing $50 \mu \mathrm{L}$ droplets of G2 media.

\section{Assessment of embryo survival and the success of embryo transfer and pregnancy}

Approximately 17 to 18 hours after warming, the morphology and blastocoel expansion of embryos was assessed using a dissecting microscope. Only expanded blastocysts with an 


\section{Obstetrics \& Gynecology Science}

Vol. 56, No. 3, 2013

Table 2. Survival and pregnancy rates of AH and no-AH treated embryos after vitrification-warming using conventional straw and PNC

\begin{tabular}{|c|c|c|c|c|c|c|}
\hline & \multicolumn{2}{|c|}{ Straw-BL } & \multicolumn{2}{|c|}{ PNC-BL } & \multicolumn{2}{|c|}{ PNC-CL } \\
\hline & AH & No-AH & $\mathrm{AH}$ & No-AH & $\mathrm{AH}$ & No-AH \\
\hline No. of vitrification-warming cycles & 11 & 16 & 7 & 5 & 50 & 28 \\
\hline No. of vitrified-warmed embryo & 31 & 51 & 29 & 12 & 321 & 197 \\
\hline No. of embryos after warming (\%) & 31 & 51 & 29 & 12 & $321(100)$ & $197(100)$ \\
\hline \multicolumn{7}{|l|}{ Embryo development after $17-18$ hours culture } \\
\hline No. of IE after warming (\%) & $26(83.9)$ & $37(72.5)$ & $20(69.0)$ & $11(91.7)$ & $179(55.8)$ & $137(69.5)^{\mathrm{a})}$ \\
\hline No. of PL embryos (\%) & & & & & $131(40.8)$ & $52(26.4)$ \\
\hline No. of survival embryos (IE+PL) (\%) & $26(83.9)$ & $37(72.5)$ & $20(69.0)$ & $11(91.7)$ & $310(96.6)$ & $189(95.9)$ \\
\hline No. of transferred embryos (mean \pm SD, range) & $\begin{array}{c}26 \\
(2.4 \pm 1.0)\end{array}$ & $\begin{array}{c}36 \\
\left(2.3^{ \pm 1.1}\right)\end{array}$ & $\begin{array}{c}18 \\
(2.6 \pm 0.5)\end{array}$ & $\begin{array}{c}11 \\
(2.2 \pm 0.8)\end{array}$ & $\begin{array}{c}227 \\
(4.5 \pm 1.9)\end{array}$ & $\begin{array}{c}122 \\
(4.4 \pm 1.5)\end{array}$ \\
\hline No. of clinical pregnancies (\% pregnancies/transfer) & $4(36.4)$ & $8(50.0)$ & $5(71.4)$ & $4(80.0)$ & $25(50.0)$ & $12(43.0)$ \\
\hline
\end{tabular}

Although 88 blastocysts of 30 cycles in straw-BL was vitrified and warmed, 6 blastocysts of 3 cycles represented severely lysed. Therefore, 6 severely lysed blastocysts were not transferred.

$\mathrm{AH}$, assisted hatching; PNC, pull and cut straw; Straw-BL, blastocyst vitrification with a conventional straw; PNC-BL, blastocyst vitrification with PNC; PNC-CL, cleaved embryo vitrification with PNC; SD, standard deviation; IE, intact embryos; PL, partial lysed; ET, embryo transfer. Values with different superscripts $\left({ }^{(a)}\right)$ differ significantly $(P<0.05)$.

intact inner cell mass and trophectoderm and embryos which looked morphologically normal were judged to have survived. Female patients were administered transdermal E2 (Estrana, 0.4 mg/day; Hisamitsu, Tokyo, Japan) with GnRH agonists for preparation of the endometrium. Progesterone was administered (50 mg in oil, daily) when the endometrial thickness reached $8 \mathrm{~mm}$. Two or three surviving blastocysts were transferred into uterus after 6 days of progesterone treatment and 3 to 5 cleaved embryos were transferred into the uterus after 4 days of progesterone treatment. Pregnancy was initially assessed by measuring serum hCG levels 14 days after embryo and blastocyst transfer. Clinical pregnancy was confirmed by the presence of fetal heart activity 30 days after embryo transfer using ultrasonography.

\section{Statistical analysis}

Data were statistically analyzed using the generalized linear model of the Statistical Analysis System (SPSS ver. 17.0, SPSS Inc., Chicago, IL, USA), analysis of variance and chi-square test. Significance was determined using a Tukey's multiple range test and $P<0.05$ was considered significant.

\section{Results}

In Table 1, data was collected from a total of 120 patients undergoing 117 treatment cycles (6 blastocysts of 3 cycles in straw group represented severely lysed. Therefore, embryo transfer of 3 cycles in straw group was not conducted) consisting of 27 cycles using blastocyst vitrification with a conventional straw (straw-BL group); 12 cycles using blastocyst vitrification with PNC (PNC-BL group); and 78 cycles using cleaved embryo vitrification with PNC (PNC-CL group). The mean maternal age for straw-BL was $31.3 \pm 3.8$ years (range, 24 to 38 years).

The number of vitrified blastocysts in the straw-BL and PNC groups was 88 and 41, respectively. The number of vitrified cleaved embryos was 518 . The recovery rates of embryos from each of the groups were $100 \%$. The survival rates of embryos after warming from straw-BL, PNC-BL, and PNC-CL groups were $71.6 \%, 75.6 \%$ and $61 \%$ respectively. However, in the case of including survival rate of partial lysed embryos (35.3\%) in PNC-CL group, the survival rate was $96.3 \%$. The pregnancy rate of the PNC-BL group (75\%) was no statically different than that of the straw-BL (44.4\%) and the PNC-CL (47.4\%) groups.

AH treatment was performed on poor quality blastocysts and cleaved embryos that exhibited zona hardening and/or partial lysis after vitrification during their development. Embryos were separated into two groups termed no-AH (good quality embryos without assisted hatching) and $\mathrm{AH}$ (poor quality embryos with assisted hatching). The survival rates of 


\section{Obstetrics \& Gynecology Science}

Joon Gyo Lim, et al. Freezing and assisted hatching of embryo

$\mathrm{AH}$ and no $\mathrm{AH}$ treated in all groups were not statically different (Table 2).

A comparison of the rates of survival after warming and the rates of clinical pregnancy between the $\mathrm{AH}$ and no-AH groups in blastocysts and cleaved embryos was performed (Table 2). Since AH treatment was only performed on poor quality cleaved embryos, the developmental rate of no-AH embryos was significantly higher than that of $\mathrm{AH}$ embryos in the cleaved embryo group (69.5\% and $55.8 \%$, respectively) with a lower rate of partial lysis $(26.4 \%$ and $40.8 \%$, respectively). No significant difference in the rate of clinical or ongoing pregnancy was observed between the no-AH blastocyst group and the AH blastocyst group. Additionally, we observed no significant difference in the rate of clinical or ongoing pregnancy between the no-AH cleaved embryo group and the AH cleaved embryo group ( $43 \%$ and $50 \%$, respectively) using PNC method. This result indicates that AH treatment of poor quality (partial lysed) embryos may raise pregnancy rate to a similar level of that obtained with untreated good quality embryos.

\section{Discussion}

Our results show that the pregnancy rate was not different in the PNC-BL group and straw-BL group. The PNC container was designed to reduce the volume of cryoprotectant needed and to simplify the vitrification procedure. We found that this container successfully cryopreserved both cleaved embryos and blastocysts. The PNC device can be easily constructed in the laboratory at a significantly lower cost (approximately $\$ 0.5$ [USD] per device) than commercial kits (cryoloops cost approximately $\$ 20$ [USD] per container). Furthermore, the PNC has a fast cooling speed with a small volume of loaded solution. The cooling rate in a $0.25 \mathrm{~mL}$ straw is $2,000^{\circ} \mathrm{Cl}$ min [15]. However, the cooling rate of cut standard straw (CSS), original form of PNC, is calculated to be $>20,000^{\circ} \mathrm{C} /$ min and also yields high oocyte survival rates after warming [3]. Although we did not measured the cooling speed of PNC, the PNC container has a thinner wall than the original CSS container and contains less vitrification solution, meaning the cooling speed of PNC may be faster than that of CSS. An increase in the rate of cooling may help reduce freezing damage and the cryoprotectant concentration, resulting in a higher survival rate after warming and an increase in the clinical pregnancy rate. The clinical outcomes resulting from the transfer of cleaved embryos and blastocysts vitrified using PNC were comparable to those of embryos vitrified using other methods $[16,17]$. Therefore, PNC may be the most costefficient and easily available vitrification method for embryos at various developmental stages.

Although there have been dramatic developments in IVF and ICSI techniques, some euploid embryos with full developmental potential still fail to implant due to hatching difficulties [18]. Spatially, in frozen-warmed embryos, cryopreservation and/or the freeze-warm process is thought to induce alterations in the glycoprotein matrix, which results in zona hardening [19]. Although $\mathrm{AH}$ has been developed to improve the rate of implantation of cryopreserved embryos, the benefits of $\mathrm{AH}$ are still debatable. To study the effects of $\mathrm{AH}$, we investigated the rates of embryo survival, partial lysis and pregnancy in $\mathrm{AH}$ and no-AH groups. In Table 2, AH was performed on 356 poor quality cleaved embryos and blastocysts. We compared the survival and pregnancy rates of blastocysts and cleaved embryos in $\mathrm{AH}$ and no-AH groups. Warming did not result in a significant difference in the survival rates between $\mathrm{AH}$ blastoctyes and no-AH blastocytes (Table 2). When blastocyst vitrification was performed, the clinical pregnancy rate of the no-AH group was not different from that of the AH group. It is well studied that survival and clinical pregnancy rates of partial lysed embryos were inferior than that of fresh and normal embryo $[8,11]$. However, in our study, AH treatment of poor quality embryos such as partial lysed embryos increased the pregnancy rate to a similar level of good quality embryo group (no-AH) (Table 2).

There are several explanations for the beneficial effects of $\mathrm{AH}$ on pregnancy rates. Firstly, $\mathrm{AH}$ may help synchronize the implantation window, a critical period during which the endometrium is ideal for implantation, with embryo hatching. Ideally, embryo hatching and the implantation window should coincide to maximize successful implantation [20]. Weakening within the zona pellucida may lead to embryos hatching earlier than normal, which could allow embryos to contact the endometrium before the implantation window [21]. Secondly, many molecules can pass through the zona pellucida and it is possible that $\mathrm{AH}$ treatment facilitates this movement. The thickness of the zona may affect the rate of transport of various molecules and nutrients such as growth factors. The presence of the artificial gap generated by AH treatment may facilitate the transport of metabolites and growth factors across 


\title{
Obstetrics \& Gynecology Science
}

\author{
Vol. 56, No. 3, 2013
}

the zona pellucida [6], which in turn may enhance embryo development and blastocyst formation [22]. Thirdly, blastocysts require a certain level of physical strength to hatch. A previous study reported that embryos with less physical strength, such as poorly developed and/or partial lysed embryos, are unable to overcome the rigidity of the zona pellucida and often fail to implant [23]. Artificial thinning or opening of the zona pellucida at an earlier developmental stage, or lysis of embryos due to physical damage, might increase the incidence of hatching $[13,24]$. AH treatment, which reduces the rigidity of the zona pellucida, may also help overcome this barrier and thereby improve the rate of hatching. In conclusion, selective $\mathrm{AH}$ treatment of poor quality embryos may significantly increase the hatching rate by synchronizing hatching with the implantation window; by assisting embryo development via facilitation of the passage of molecules and nutrients across the zona pellucida; and by reducing the rigidity of the zona pellucida via the introduction of artificial gaps.

Our results suggest that PNC is a simple and inexpensive procedure that can increase the number of clinical pregnancies via the vitrification of blastocysts. In addition, selective $\mathrm{AH}$ treatment of embryos that show a poor prognosis after warming may increase the rate of clinical pregnancy.

\section{Acknowledgments}

This work was supported by a grant from the Next-Generation BioGreen 21 program (\#PJ008067), Rural Development Administration, Republic of Korea.

\section{References}

1. Rall WF, Fahy GM. Ice-free cryopreservation of mouse embryos at -196 degrees $C$ by vitrification. Nature 1985;313:573-5.

2. Desai N, Blackmon H, Szeptycki J, Goldfarb J. Cryoloop vitrification of human day 3 cleavage-stage embryos: post-vitrification development, pregnancy outcomes and live births. Reprod Biomed Online 2007;14:208-13.

3. Kim HJ, Kim CH, Lee JY, Kwon JH, Hwang D, Kim KC. Effect of cryopreservation day on pregnancy outcomes in frozen-thawed blastocyst transfer. Korean J Reprod Med 2010;37:57-64.
4. Shin MR, Choi HW, Kim MK, Lee SH, Lee HS, Lim CK. In vitro development and gene expression of frozen-thawed 8-cell stage mouse embryos following slow freezing or vitrification. Clin Exp Reprod Med 2011;38:203-9.

5. Drobnis EZ, Andrew JB, Katz DF. Biophysical properties of the zona pellucida measured by capillary suction: is zona hardening a mechanical phenomenon? J Exp Zool 1988;245:206-19.

6. Cohen J, Alikani M, Trowbridge J, Rosenwaks Z. Implantation enhancement by selective assisted hatching using zona drilling of human embryos with poor prognosis. Hum Reprod 1992;7:685-91.

7. De Vos A, Van Steirteghem A. Zona hardening, zona drilling and assisted hatching: new achievements in assisted reproduction. Cells Tissues Organs 2000;166:220-7.

8. El-Toukhy T, Khalaf Y, Al-Darazi K, Andritsos V, Taylor A, Braude P. Effect of blastomere loss on the outcome of frozen embryo replacement cycles. Fertil Steril 2003;79:1106-11.

9. Tucker MJ, Cohen J, Massey JB, Mayer MP, Wiker SR, Wright G. Partial dissection of the zona pellucida of frozen-thawed human embryos may enhance blastocyst hatching, implantation, and pregnancy rates. Am J Obstet Gynecol 1991;165:341-4; discussion 4-5.

10. Check JH, Hoover L, Nazari A, O'Shaughnessy A, Summers $D$. The effect of assisted hatching on pregnancy rates after frozen embryo transfer. Fertil Steril 1996;65:254-7.

11. Nagy ZP, Taylor T, Elliott T, Massey JB, Kort HI, Shapiro DB. Removal of lysed blastomeres from frozen-thawed embryos improves implantation and pregnancy rates in frozen embryo transfer cycles. Fertil Steril 2005;84:1606-12.

12. Martins WP, Rocha IA, Ferriani RA, Nastri CO. Assisted hatching of human embryos: a systematic review and meta-analysis of randomized controlled trials. Hum Reprod Update 2011;17:438-53.

13. Schiewe MC, Hazeleger NL, Sclimenti C, Balmaceda JP. Physiological characterization of blastocyst hatching mechanisms by use of a mouse antihatching model. Fertil Steril 1995;63:288-94.

14. Lanzendorf SE, Nehchiri F, Mayer JF, Oehninger S, Muasher SJ. A prospective, randomized, double-blind study for the evaluation of assisted hatching in patients with advanced maternal age. Hum Reprod 1998;13:409-13.

15. Vanderzwalmen P, Bertin G, Debauche C, Standaert V, 


\section{Obstetrics \& Gynecology Science}

Joon Gyo Lim, et al. Freezing and assisted hatching of embryo

Bollen N, van Roosendaal E, et al. Vitrification of human blastocysts with the Hemi-Straw carrier: application of assisted hatching after thawing. Hum Reprod 2003;18:1504-11.

16. Kuwayama M, Vajta G, Kato O, Leibo SP. Highly efficient vitrification method for cryopreservation of human oocytes. Reprod Biomed Online 2005;11:300-8.

17. Mukaida T, Nakamura S, Tomiyama T, Wada S, Oka C, Kasai $M$, et al. Vitrification of human blastocysts using cryoloops: clinical outcome of 223 cycles. Hum Reprod 2003;18:384-91.

18. Huisman GJ, Fauser BC, Eijkemans MJ, Pieters MH. Implantation rates after in vitro fertilization and transfer of a maximum of two embryos that have undergone three to five days of culture. Fertil Steril 2000;73:117-22.

19. Carroll J, Depypere H, Matthews CD. Freeze-thaw-induced changes of the zona pellucida explains decreased rates of fertilization in frozen-thawed mouse oocytes. J Reprod Fertil 1990;90:547-53.

20. Liu HC, Cohen J, Alikani M, Noyes N, Rosenwaks Z. Assisted hatching facilitates earlier implantation. Fertil Steril 1993;60:871-5.

21. Malter HE, Cohen J. Blastocyst formation and hatching in vitro following zona drilling of mouse and human embryos. Gamete Res 1989;24:67-80.

22. Hershlag A, Feng HL. Effect of prefreeze assisted hatching on postthaw survival of mouse embryos. Fertil Steril 2005;84:1752-4.

23. Cohen J, Inge KL, Suzman M, Wiker SR, Wright G. Videocinematography of fresh and cryopreserved embryos: a retrospective analysis of embryonic morphology and implantation. Fertil Steril 1989;51:820-7.

24. Cohen J. Assisted hatching of human embryos. J In Vitro Fert Embryo Transf 1991;8:179-90. 